\title{
La preparación de los docentes para la dirección del aprendizaje de la producción de textos escritos
}

\author{
María del Carmen Hernández Valdés ${ }^{1}$ \\ Elizabeth Pérez Díaz ${ }^{2}$ \\ Maité Felicia Valdivia Valdés ${ }^{3}$
}

\section{Resumen}

Esta investigación fue orientada a la preparación de los docentes para la dirección de aprendizajes en la producción de textos escritos para la escuela primaria de la escuela "José Martí" del municipio La Sierpe, Cuba. Fueron propuestas que integran de forma coherente el desempeño participativo de los docentes, la autoevaluación como fuente de transformación y la participación de los agentes educativos. Estas han de orientar la dirección educativa de la producción de textos escritos, que ha de contribuir a un aprendizaje superior expresado en el alcance de los fines, principios y objetivos de la escuela primaria. El impacto esperado: Elevar la preparación de los docentes del primer ciclo de la escuela primaria para dirigir el proceso de producción textual, empleando métodos del nivel teórico y empíricos.

Palabras clave: Producción de textos escritos; actividades metodológicas; docentes.

\section{Summary}

This research was oriented to the preparation of teachers for the direction of learning in the production of written texts in elementary school. They were proposals that integrate coherently the participative performance of the educators, self-evaluation as a source of transformation and participation of the educational agents. These proposals should guide the educational direction of the written texts production, which also should contribute to higher learning expressed in the scope of the aims, principles and objectives of the primary school. The expected impact: To raise the preparation of the first cycle of primary school teachers to direct the process of textual production, using theoretical and empirical methods.

Key Words: Written texts production; methodological activities; teachers.

1 Master en Ciencias de la Educación. Profesora y coordinadora de la Carrera Maestro Primario de la Universidad “José Martí Pérez" Sancti Spíritus. Correo: mchernandez@uniss.edu.cu

2 Licenciada en Estudios Socioculturales. Profesora y coordinadora de Ciencia y Técnica de la Universidad “José Martí Pérez" Sancti Spíritus. Correo : elizabethpd@uniss.edu.cu

3 Master en Ciencias de la Educación. Suddirectora docente de la Universidad "José Martí Pérez" Sancti Spíritus. Correo: maite@uniss.edu.cu

Recibido: 05/10/2017 Aprobado: 23/11/2017 


\section{Introducción}

La Revolución Cubana marcó un viraje histórico en el movimiento revolucionario y libertador de América Latina y abrió una nueva era en la historia del pueblo cubano. Un destacado logro de Cuba socialista es la realización de la Revolución Cultural. Los cambios colosales en el campo de la educación y la preparación de los docentes confirman el significado histórico respecto a que la revolución cultural es parte inseparable de las transformaciones sociales.

La educación en el mundo actual necesita ser cada vez más eficiente. Este es uno de los grandes retos de la época contemporánea. Llevar una educación de calidad es una de las más preciadas aspiraciones de la humanidad. Desde cada aula hay que cultivar la inteligencia y los valores a través de la enseñanza. En medio de la cotidianeidad de la escuela hay que educar en los más avanzados adelantos de la ciencia y tecnología, y facilitar los esfuerzos hacia la creatividad y la independencia.

Inmersa en estos desafíos se encuentra la Educación Primaria que tiene como fin: "Contribuir a la formación integral de la personalidad del escolar, fomentando desde los primeros grados la interiorización de conocimientos y orientaciones valorativas que se reflejen gradualmente en sus sentimientos, formas de pensar y comportamiento, acorde con el sistema de valores e ideales de la Revolución socialista cubana" (Rico Montero, 200o. En esta se prioriza el perfeccionamiento del proceso docente-educativo y en especial la superación de los docentes para dirigir las acciones educativas con mayor efectividad.

En la contribución al logro de estos propósitos la producción de textos escritos por los escolares es un aspecto de suma importancia; en tanto su fin primordial es que adquieran un medio idóneo de comunicación. Además, las exigencias del mundo moderno exigen al hombre el dominio de la escritura, que se expresa en todos los ámbitos de la actividad humana. El compromiso social de los docentes es lograr que los escolares se expresen con claridad en cualesquiera situaciones de comunicación.

La dirección del proceso de enseñanza - aprendizaje de la producción de textos escritos ha sido preocupación de estudiosos de diferentes disciplinas que han tratado de buscar diferentes vías y alternativas para que los docentes puedan dirigir su desarrollo. La tradición pedagógica cubana ha sido rica en apreciaciones y aportes sobre la problemática que se aborda. En la primera mitad del siglo XX, se destacan los trabajos de Carolina Poncet y de Cárdenas (1879-1969) y de Alfredo Miguel Aguayo (1866-1948); en la segunda mitad las obras de Herminio Almendros (1898-1974), Ernesto García Alzola (1978), Delfina García Pers (1983) y Georgina Arias Leyva (2003), quienes fueron portadores de una didáctica dirigida al desarrollo de la lengua materna en la escuela primaria. 


\section{EDUCACIÓN}

En las últimas décadas del siglo XX y los primeros años del siglo XXI el tema de la producción de textos escritos ha sido tratado por diversos investigadores, entre los que destacan Magalys Ruiz (1996) y Angelina Roméu Escobar (1997), quienes, a partir del desarrollo de la lingüística textual, de la teoría de la comunicación y del auge del enfoque comunicativo, han sistematizado estudios que constituyen antecedentes de obligada consulta.

En el territorio de Cuba se han desarrollado investigaciones que particularizan en diferentes aristas de la temática, entre ellas destaca la tesis de doctorado de María de los Ángeles García Valero (2005), cuyos aportes han resultado de gran valor en la presente investigación y que aborda el tema de la producción verbal de textos escritos en los escolares primarios. Así mismo, existen diferentes propuestas en las que se aborda la preparación del docente y la labor de los jefes de ciclo desde diferentes aristas, entre las cuales destacan Aida Chinea Campos (2007).

Las propuestas anteriores, reafirman la necesidad y posibilidad del desarrollo de estudios ajustados a contextos particulares, en los que se manifiestan limitaciones que apuntan a la presencia de las siguientes manifestaciones:

- Se trabaja en todas las clases la lectura de textos y su comprensión, pero no se realiza un análisis de los textos que se leen con el objetivo de que los escolares comprendan cómo se estructuran con vista a que puedan servirles de modelos constructivos.

- No se concibe en los sistemas de clases tareas relacionadas con la búsqueda de la información como preparación del escolar para escribir.

- Para la concepción de los sistemas de clases no se seleccionan las lecturas que amplíen la competencia cultural de los escolares en la temática seleccionada.

- No se elaboran tareas docentes en forma de situación de comunicación para lograr en los escolares una verdadera motivación para escribir.

- No se emplean las tecnologías de la informática y las especialidades como una vía para optimizar el proceso educativo de la producción de textos escritos.

En virtud de satisfacer esas necesidades se determina como objetivo: proponer actividades metodológicas que contribuyan a elevar la preparación de los docentes de primer ciclo de la escuela primaria para la dirección del aprendizaje de la producción de textos escritos; para lo cual se aplicaron los siguientes métodos científicos: histórico y lógico, análisis y síntesis, inducción y deducción, modelación, enfoque de sistema, encuesta, observación, análisis de documentos y experimento pedagógico.

Se considera como elemento novedoso, desde un accionar metodológico, la conjugación participativa de los docentes, la autoevaluación como vía para impulsar la transformación de los sujetos y la implicación de los agentes educativos que desde el cumplimiento de sus funciones contribuyen a optimizar el proceso de producción de textos en el primer ciclo de la escuela primaria. 


\section{Revisión de literatura}

En el proceso histórico, distinguidos pedagogos e investigadores se han dedicado a estudiar las vías de preparación de los docentes para enfrentar con calidad el proceso docente educativo. A partir del triunfo de la Revolución el trabajo metodológico comenzó a alcanzar la verdadera dimensión y esencia como vía para la preparación metodológica de los docentes y desde los inicios de la década del 70 del siglo XX la actividad metodológica empieza a cobrar mayor fuerza al considerársele una vía efectiva para orientar a los docentes en el trabajo técnico-docente.

El Plan de Perfeccionamiento del Sistema Nacional de Educación se implantó en el período 1975-1981 y el trabajo metodológico se dedicó fundamentalmente a la elaboración de los métodos y contenidos de la enseñanza y a las actividades encaminadas a capacitar a docentes y profesores en estos componentes educativos. Luego, entre1982-1992, se buscó el perfeccionamiento del trabajo educativo en los centros y tuvo como objetivos fundamentales lograr que el personal docente aprendiera a desarrollar eficientemente su labor profesional y a valorar la efectividad de esta tarea por los resultados obtenidos en los escolares en el desarrollo de capacidades, habilidades y hábitos.

El término trabajo metodológico no se reduce al proceso de enseñanza- aprendizaje ni se limita a la relación docente-alumno, sino que abarca también la formación integral del docente como ser humano, como ciudadano comprometido con el futuro del país y como profesional, lo cual exige enseñarlo a enseñar, enseñarlo a educar y educarlo para que pueda cumplir con éxito, su misión educativa: la acertada conducción del Proceso Docente - Educativo

En esta tesis la autora acepta como Proceso Docente - Educativo la acepción de Álvarez Carlos (1996), quien lo conceptualiza:

“(...) aquel, que como resultado de las relaciones sociales que se dan entre los sujetos que en él participan, está dirigido de un modo sistémico y eficiente, a la formación de las nuevas generaciones, tanto en el plano educativo como instructivo y desarrollador (objetivo); con vista a la solución del encargo social (problema), mediante la apropiación de la cultura que ha acopiado la humanidad en su desarrollo (contenido); a través de la participación activa y consciente de los estudiantes (métodos); planificada en el tiempo y observando ciertas estructuras organizativas estudiantiles (forma); y con ayuda de ciertos objetos (medios); a través de lo cual se obtienen determinadas consecuencias (resultados); y cuyo movimiento está determinado por las relaciones causales entre esos componentes y de ellos con la sociedad (leyes) que constituyen su esencia." (27: 24). 


\section{EDUCACIÓN}

De acuerdo con la definición anterior, este proceso parte de una relación con el contexto social, con la vida, estableciendo el movimiento entre componentes y sus relaciones, permitiendo abordar la instrucción y la educación como sistema en el contexto de la escuela. El trabajo metodológico deberá constituir la vía principal en la preparación de los docentes para lograr que pueda concretarse, de forma integral, el sistema de influencias que ejercen en la formación de sus escolares para dar cumplimiento a las direcciones principales del trabajo educacional y las prioridades de las enseñanzas.

En los documentos normativos, el trabajo metodológico, se evidencia como elemento intrínseco al desarrollo del proceso docente - educativo en una escuela, y su calidad depende de la eficiencia con que se organice y ejecute el sistema de trabajo metodológico del centro o colectivo pedagógico, es la actividad sistemática y permanente de los docentes, encaminada a mantener y elevar la calidad del proceso docente - educativo mediante el incremento de la maestría pedagógica de los docentes, el desarrollo o confección de los medios de enseñanza, la determinación de los métodos de enseñanza, la evaluación del aprendizaje y demás aspectos que aseguren el proceso docente - educativo.

$\mathrm{Al}$ analizar estos conceptos se puede apreciar que existen elementos comunes. Estos resultan de gran importancia para poder conducir el trabajo metodológico en la escuela: Es una vía para elevar la calidad del proceso pedagógico, son actividades sistemáticas, creadoras, intelectuales para la preparación de docentes a fin de garantizar el cumplimiento de las principales direcciones educacionales y contribuye a la superación de los docentes.

Los docentes deben ser capaces de promover el desarrollo intelectual de los escolares, de atenderlos individualmente en conformidad con sus necesidades y de lograr desarrollar una enseñanza diferenciada que asegure a cada uno de ellos un máximo desarrollo según sus posibilidades, para ello hay que tener también en cuenta los aspectos formativos, es preciso trabajar por el desarrollo integral de la personalidad para la formación integral de las nuevas generaciones. La elevación de la eficiencia docente y educativa es la respuesta a las necesidades actuales de nuestra sociedad.

El aporte fundamental de la asignatura Lengua Española consiste esencialmente en el desarrollo de la capacidad de comunicación en forma oral y escrita, en la contribución a la formación y expresión del pensamiento, así como en la adquisición inicial y posibilidad de disfrute de la belleza artística y del caudal de conocimientos que ha atesorado la humanidad gracias a la palabra.

La enseñanza de nuestra lengua materna tiene como objetivo fundamental dotar a los educandos del instrumento que les permita comunicarse amplia y acertadamente en el ámbito social, tanto en forma oral como escrita, así como asimilar 
las diferentes asignaturas que participan en su formación multilateral y armónica, preparándolos para la actividad laboral, colectiva y creadora, todo lo cual demuestra el carácter instrumental de esta materia para que cumpla importantes funciones tales como proporcionar el intercambio humano, organización, desarrollo y expresión del pensamiento y la regulación de la conducta, el intercambio y transformación social.

Es en la escuela donde el alumno adquiere el instrumento de la lectura-escritura y se establece la adecuada correspondencia entre la lengua oral y escrita, de este modo se establecen desde el primer momento los vínculos entre la operación decodificadora de leer y la operación codificadora de escribir y la relación dialéctica que existe entre ambas.

El enfoque metodológico de la asignatura Lengua Española, en la Educación Primaria es eminentemente práctico; es el punto de partida para arribar a la teoría y que esta permita volver a una práctica enriquecida. Tendrá como eje fundamental la producción verbal, es decir el desarrollo de la expresión oral y escrita a partir de las impresiones y motivaciones que la vida provoca en sus múltiples aspectos.

La didáctica de la producción de textos escritos ha sido preocupación constante de estudiosos que han tratado de buscar diferentes vías y alternativas para que los docentes puedan dirigir acertadamente su desarrollo. En distintas épocas, los pedagogos cubanos se han referido a este importante tema y han aportado luz a esta enseñanza. La obra de la doctora Delfina García Pers sobresale al ofrecer orientaciones sobre cómo desarrollar la lengua escrita en la escuela primaria y sobre cómo concebir el enfoque comunicativo en la construcción de textos como una práctica que prepare al niño para la vida a través de la escritura de distintos tipos de textos con diferentes intenciones comunicativas. Su obra: Didáctica del idioma español (1978), ha servido durante años a todos los docentes para llevar a feliz término, la enseñanza de la Lengua Española.

El poeta y pedagogo Raúl Ferrer Pérez también se refirió a este tema. Desde su dirección se redactó una ponencia acerca de la enseñanza de nuestro idioma y sus principales problemas. En esta se compendian problemas como la insuficiente preparación cultural, pedagógica y lingüística de algunos docentes; la subestimación de la influencia de los primeros grados en el desarrollo de las habilidades de expresión y comprensión de los escolares; la inadecuada selección de métodos y procedimientos para la enseñanza del idioma y la ausencia de medios de enseñanza, entre otras. Planteaba que: "Defender la calidad del lenguaje es defender la calidad de la enseñanza". (Ferrer Pérez, R., 1980:93)

Por su parte, la destacada pedagoga cubana Angelina Roméu Escobar (1990, 1995, 1996) propone una metodología con enfoque comunicativo para la enseñanza de la Lengua Española a partir de los tres componentes funcionales de la asignatura en el nivel medio: comprensión, análisis y construcción de textos. Le concede gran 


\section{EDUCACIÓN}

importancia al trabajo oral antes de la escritura como una forma de explorar los conocimientos de los escolares sobre la temática, de ampliar sus ideas y coincide en que el proceso de producción de textos escritos transita por tres etapas: orientación, ejecución y control. Esta autora no hace énfasis en la forma en que el docente primario debe organizar su análisis metodológico para la planificación del sistema de clases.

La obra de estos pedagogos enfatiza de forma general, la necesidad de potenciar la expresión oral como base para el desarrollo de la expresión escrita, en el uso de diferentes técnicas para enseñar adecuadamente a los escolares a corregir sus textos. Además, destacan la importancia de desarrollar este proceso en un ambiente favorable.

Dentro de las orientaciones más actuales que norman el trabajo con la producción de textos escritos en la enseñanza primaria se encuentran las de la doctora Georgina Arias Leyva (2003) que ha planteado que para lograr que los escolares se expresen por escrito de forma adecuada es imprescindible que se creen situaciones reales de expresión y comunicación, considerando las fases que se deben cumplir en este proceso: la pre-escritura, la escritura y la reescritura.

Por su parte, María de los Ángeles García Valero $(1998,2005)$ ha realizado una amplia investigación sobre el desarrollo de la producción verbal de textos escritos por los escolares primarios. Esta autora plantea que:

el docente realizará un análisis metodológico de los objetivos y contenidos de todas las asignaturas para conocer qué tipos de textos deberán escribir los escolares en un período de clases o en el curso escolar y en qué asignaturas. Esto les permitirá planificar el sistema de clases de Lengua Española de esa unidad o período y darle a cada texto su adecuado tratamiento metodológico.

Ligia Sales Garrido (2007:19), afirma que la aplicación del enfoque cognitivo, comunicativo y sociocultural puede constituir un recurso metodológico para que la enseñanza de la Lengua Española contribuya al desarrollo de la competencia comunicativa de los escolares de la Educación Primaria a partir de su concepción teórica y metodológica.

Plantea que de acuerdo con la situación comunicativa se construye el texto que se corresponda con esta, por tanto, "es necesario que se tenga dominio de las características fundamentales de cada tipo de texto, esto facilita el orden lógico, la coherencia y la suficiencia, aspectos que permiten una construcción textual coherente con carácter personológico, comunicativo, social, pragmático estructurado y cierre semántico" (Sales Garrido, L., 2007:21)

Los trabajos realizados por M. Georgina Arias Leyva, María de los Ángeles García Valero y Ligia Sales Garrido presentan gran actualidad, valor teórico y práctico, se 
corresponden plenamente con la política actual de nuestro sistema educacional, son un modelo para el trabajo metodológico y ofrecen de forma clara y científica un modo de proceder para la dirección del aprendizaje de la producción de textos escritos en la escuela primaria.

\section{Materiales y métodos}

El trabajo fue realizado a partir de la aplicación del método de experimento pedagógico, en su modalidad de pre - experimento, con un diseño de pre - test y pos - test, con control de la variable dependiente: nivel de preparación metodológica de los docentes del primer ciclo para la dirección del aprendizaje de la producción de textos escritos.

A partir de la operacionalización de la variable se determinaron como indicadores:

1. Nivel de conocimiento de los tipos de textos y de las características que los distinguen.

2. Nivel de dominio de las exigencias didácticas para concebir las tareas a partir de una situación comunicativa.

3. Nivel en que logra la concepción de las tareas de aprendizaje para la producción de textos, con ajuste a las exigencias didácticas de la situación comunicativa.

4. Nivel de dominio de la metodología para el tratamiento a la producción de textos.

Teniendo en cuenta los indicadores, se seleccionaron los métodos fundamentales para determinar el estado de la variable dependiente antes (pre - test) y después (post - test) de la introducción de la variable independiente. Los métodos utilizados en ambos momentos fueron: la prueba pedagógica (pre - test y pos - test), y el análisis de documentos.

El análisis de la situación inicial de la preparación de los docentes del primer ciclo de la escuela "José Martí" del municipio La Sierpe, Cuba para el logro de estos propósitos se realizó a partir de la aplicación de un conjunto de métodos e instrumentos que abarcó la revisión de programas, orientaciones metodológicas, adecuaciones curriculares y un grupo de sistemas de clases planificadas por los docentes y entrevistas, que hicieron posible determinar regularidades en la situación real de la dirección del aprendizaje de la producción de textos escritos.

Es evidente que en el tema que ocupa a esta investigación existen aún insuficiencias en la actuación pedagógica de los docentes investigados, corroborándose en deficiencias en la aplicación de los fundamentos básicos que rigen la enseñanza-aprendizaje de la producción de textos escritos, obstaculizando el desarrollo adecuado de este componente en los escolares. 
Durante el estudio inicial realizado, se confirma la necesidad de elaborar actividades metodológicas dirigidas a la preparación de los docentes del primer ciclo de la escuela primaria José Martí en la dirección del aprendizaje de la producción de textos escritos, pues los estudios realizados no han dado respuesta a esta problemática.

\section{Resultados y discusión}

Después de constatar, mediante el diagnóstico realizado, la problemática existente en relación con la preparación metodológica de los docentes del primer ciclo de la escuela primaria "José Martí" para la dirección del aprendizaje de la producción de textos escritos y, en correspondencia con el objetivo de esta investigación, se realiza una propuesta de actividades metodológicas, sobre la base de los presupuestos teóricos que la sustentan.

La planificación deberá enfocarse en: trabajo con modelos que permitan a los escolares reconocer las características de los textos y realizar reflexiones sobre la lengua, búsqueda y procesamiento de la información, ampliación del vocabulario, actividades de expresión creadora que les permitan desarrollar la imaginación, la creatividad y expresar sus sentimientos y emociones, entre las que no deben faltar actividades lúdicas y de expresión plástica, el trabajo con textos borradores que faciliten el proceso de enriquecimiento y perfeccionamiento de lo que se escribe, la escritura de la versión final y la publicación de las producciones escritas.

Estos elementos conducen a plantear que el docente debe preverlo en su análisis metodológico y organizar un sistema de tareas que favorezcan la adquisición de esta competencia cultural. Esto debe hacerse con dos objetivos: ampliar la competencia cultural de los escolares en relación con la temática seleccionada para escribir y lograr la formación del hábito de consultar diferentes fuentes para poder escribir con propiedad sobre una temática determinada.

Se considera que otro elemento importante lo constituye las actividades de lecturas que realizan en el aula y en la biblioteca escolar, la observación de vídeos y películas relacionadas con la temática sobre la que se desean escribir y el trabajo con los softwares educativos, así como la creación y apreciación de obras plásticas referidas al tema seleccionado desde los talleres de creación que dirige el instructor de arte. De manera que cuando se dispongan a escribir puedan hacerlo con un arsenal suficiente de conocimientos y con un grupo de anotaciones que les permita trabajar y crear. Trabajar con esta concepción integradora garantiza una mayor eficiencia en la dirección del proceso de enseñanza-aprendizaje de la producción de textos escritos.

Estas actividades se realizarán en todos los sistemas de clases que los docentes planifiquen durante el curso escolar, las cuales permitirán el trabajo con cualquier tipo de texto que se pretenda producir. Tendrán como núcleo integrador la asignatura 
Lengua Española, pero en las fases para el desarrollo de la producción oral y escrita se realizará, además, en aquellas asignaturas y procesos que ocurran en la escuela donde se esté abordando la temática del tema que se escribirá.

El proceso de construir un texto no puede ser visto solamente de esa manera. La planificación, la textualización y la revisión son procesos recursivos y en la concreción del acto escritural se recurre a ellos en cualquier momento. Por lo que hay que verlos desde una doble perspectiva: como aprendizaje en sí mismo de un contenido procedimental, y como medios de adquisición metalingüísticos que le permitan al niño las reflexiones sobre la lengua.

Debe considerarse, además de la preparación de asignatura, la participación del docente, el profesor de computación, el instructor de arte y bibliotecaria. Los elementos que siguen, para el logro de estos propósitos:

- Planeación de la escritura, para lo cual el docente debe utilizar diferentes métodos y procedimientos que le permitan al escolar desarrollar habilidades para buscar la información y organizarla en correspondencia con el tipo de texto que va a escribir.

- Presentar tareas en forma de situación comunicativa, de manera que puedan identificar los posibles receptores que tendrán los trabajos escritos y que el proceso tenga sentido y significado para los escolares. Esto lleva a que logren la verdadera motivación para escribir.

- Seleccionar lecturas que sirvan de modelos constructivos en las cuales los escolares reconocerán las restricciones del tipo de texto y el empleo de las diferentes estructuras lingüísticas.

- Seleccionar las lecturas que se trabajarán en la unidad con el propósito de que los escolares amplíen la competencia cultural en la temática seleccionada.

- Organizar el sistema de tareas investigativas que debe orientar para la obtención de la competencia cultural de los escolares, requisito indispensable del acto de escritura.

- Seleccionar los temas que a otras asignaturas se correspondan estableciendo el vínculo interdisciplinario de manera que el escolar pueda recopilar la información que necesita a través de las diferentes estrategias de organización de la información.

- Utilizar los vídeos y las películas que se relacionen con la ampliación de la competencia cultural de los escolares.

- Concebir creaciones y apreciaciones plásticas relacionadas con la temática sobre la que van a escribir en los talleres de creación, con previa coordinación con el Instructor de Arte. 


\section{EDUCACIÓN}

- Establecer coordinaciones con la biblioteca escolar para que las actividades estén relacionadas con el tema y el tipo de texto que se trabajará en las aulas, así como para la disponibilidad y localización de la bibliografía que los escolares necesitan consultar.

- Ilustrar el texto con los dibujos realizados o con los recortes recopilados.

- La utilización del procesador de textos Word, no como parte del programa de computación, sino como parte inseparable de la asignatura Lengua Española y del tratamiento metodológico para la dirección de su aprendizaje.

- Empleo de textos borradores que les permitan tachar, borrar, volver a escribir hasta lograr el perfeccionamiento del texto, pero para ello es necesario que el docente les demuestre la utilidad de su uso y cómo pueden mejorar el estilo escribiendo diferentes versiones, que ofrezcan mayor información y una organización más pertinente.

Se considera que la construcción de textos es un proceso que exige capacidad para seleccionar y emplear adecuadamente los medios lingüísticos en correspondencia con una intención, una finalidad y una necesidad de significar del sujeto, en una situación comunicativa determinada y en un contexto sociocultural específico.

\section{Actividades Metodológicas}

La dirección del aprendizaje de la producción de textos escritos que se proponen, se distinguen por las siguientes características generales:

1 El rol participativo de los docentes.

2 La autoevaluación como fuente de transformación.

3 La participación de los agentes educativos (profesor de computación, instructor de arte y bibliotecaria).

Cada uno de las actividades metodológicas se estructuraron del siguiente modo: título, objetivo, sesión de inicio, discusión, evaluación, preparación para la próxima actividad y el cierre de la actividad. Son estos aspectos determinados por Calero (2005) en su tesis doctoral, las cuales se asumen en este trabajo.

La sesión de inicio persigue tres objetivos:

- Movilizar a los participantes para promover ideas variadas, con el propósito de lograr que sean lo más imaginativos y originales posibles.

- Crear un clima de libertad y espontaneidad de expresiones.

- Preparar a los directivos asistentes para la sesión de discusión.

La discusión se organiza en pequeños grupos y en sesión plenaria. Tiene como finalidad: 
- Valorar los aciertos, posibilidades y limitaciones que poseen sobre sí mismos y a través de la comunicación con otros.

- Crear ambientes donde se incrementen las expectativas de cada participante ante las interrogantes y se generen variadas alternativas para su solución.

La sesión de evaluación tiene como finalidad que:

- Cada participante se compare con otro del grupo y con el nivel de preparación refrendado en el objetivo.

- El grupo emita juicios de valor sobre la participación, incluyendo el reconocimiento del desempeño de los más destacados.

- El coordinador emite un juicio valorativo de la evaluación realizada.

En las actividades, la evaluación proviene del consenso que se establece entre el coordinador y los participantes, al finalizar cada una, se selecciona el sujeto o equipo de mejor actuación y se premian los mejores resultados, transitando por formas como la autoevaluación y la coevaluación.

La preparación para el próximo taller tiene como propósito la orientación de tareas a ejecutar por los participantes dirigidas al aseguramiento del nivel de partida para la próxima actividad. Esta sesión puede cumplirse de forma colectiva o individual y para lograr una mayor independencia en la realización de las tareas es necesario que disminuyan los niveles de ayuda de una actividad a otra, observando el principio de las exigencias decrecientes. En el cierre del taller se aplican técnicas e interrogantes dirigidas a medir el estado de satisfacción que siente la estructura de dirección en la realización de las actividades.

Después de aplicada la propuesta en la Escuela Primaria se evidencian avances sustanciales en la preparación de los docentes para la dirección del proceso de producción de textos escritos, visible en el dominio que poseen de los tipos de textos, sus características, los elementos que forman parte de una situación comunicativa, las etapas para el tratamiento a este componente, los elementos a tener en cuenta en función de ampliar la competencia cultural de los escolares en la temática seleccionada para escribir, utilizando términos propios de las ciencias pedagógicas y sistematización de criterios en la literatura especializada, asimismo con alto grado de independencia logran organizar los sistemas de clases teniendo en cuenta las exigencias planteadas. Demuestran además conocimiento de las coordinaciones que deben establecer con el propósito de optimizar el proceso de producción de textos escritos. 


\section{Conclusiones}

La preparación de los docentes del primer ciclo en la dirección de la producción de textos escritos se enfoca desde la perspectiva socio-histórico-cultural y se tiene en cuenta el desarrollo profesional y humano del docente; lo que quedó evidenciado en la revisión de los documentos que norman y orientan el tratamiento a esta problemática en la enseñanza primaria, los cuales constituyen valiosos recursos para la preparación de los docentes.

En la preparación de los docentes del primer ciclo de la escuela primaria se manifiestan insuficiencias de índole metodológica, lo que se compromete más por las limitaciones en el dominio que poseen de los tipos de textos, sus características, los elementos que forman parte de una situación comunicativa, las etapas para el tratamiento a este componente, los elementos a tener en cuenta en función de ampliar la competencia cultural de los escolares en la temática seleccionada para escribir, asimismo de cómo organizar los sistemas de clases teniendo en cuenta las exigencias planteadas y las coordinaciones que deben establecer con el propósito de optimizar el proceso de producción de textos escritos.

Las actividades que se proponen, vinculan el trabajo metodológico como vía fundamental para la preparación de los docentes, asumiendo la autoevaluación, el protagonismo de los docentes y la participación de los agentes educativos como recursos para impulsar la transformación del estado real al deseado en la preparación de los docentes del primer ciclo para la dirección del aprendizaje de la producción de textos escritos.

La validación de las actividades metodológicas mediante la aplicación del preexperimento, permitió determinar que es factible de generalizar y que se proyecta a perfeccionar la preparación de los maestros del primer ciclo en la dirección de la producción de textos escritos corroborado en la evolución favorable en los indicadores declarados.

\section{Lista de referencias}

Aguayo, A. M. (1910). Enseñanza de la lengua materna en la escuela elemental. La Habana: Librería Nueva.

Almendros, H. (1969). Guía de Español. La Habana: Editorial Pueblo y Educación.

Álvarez de Zayas, C. (1995). Metodología de la Investigación Científica. Centro de Estudios de Educación Superior "Manuel F. Gran". Universidad de Oriente. Santiago de Cuba. Impresión ligera. 
Arias Leyva, G. (2003). Hablemos sobre la comunicación escrita. Cartas al maestro 6. La Habana: ICCP.

Cancio, C. (1999). Habilidades de expresión escrita: enfoque comunicativo_motivacional. En Taller de la palabra La Habana: Editorial Pueblo y Educación.

Chinea Campo, A. (2007). Estrategia Metodológica para perfeccionar la labor del Jefe de Ciclo en el Trabajo Metodológico. Tesis en Opción del Título Académico de Máster en Ciencias de la Educación. ISP Félix Varela. Villa Clara.

Ferrer Pérez, R. et al.(1980). Consideraciones acerca de la enseñanza de nuestro idioma: sus principales problemas". En: Seminario Nacional 3. parte. Empresa de Impresoras Gráficas del MINED. La Habana.

García Alzola, E. (1975). Lengua y Literatura. La Habana: Editorial Pueblo y Educación.

García Pers, D. (1978). Didáctica del idioma español. La Habana: Editorial Pueblo y Educación.

García Valero, M. Á. (1998). Metodología para dirigir la enseñanza de la construcción de textos con un enfoque comunicativo. Tesis en opción al título de Master en Educación Primaria. La Habana: ISP Enrique José Varona.

García Valero, M. Á. (1998). Metodología para dirigir la enseñanza de la construcción de textos con un enfoque comunicativo. Tesis en opción al título de Máster en Educación Primaria. La Habana: ISP Enrique José Varona.

García Valero, M. Á. (2005). Estrategia metodológica para el desarrollo adecuado de la producción verbal de textos escritos por los escolares primarios. Tesis en opción al grado científico de Doctora en Ciencias Pedagógicas. Villa Clara: ISP "Félix Varela".

Ministerio de Educación, Cuba. (1986). Resolución Ministerial 290/86. Indicaciones para la realización del trabajo metodológico. La Habana.

Ministerio de Educación, Cuba.(2014).Resolución Ministerial 200. Reglamento del Trabajo Metodológico. La Habana.

Montaño Calcines, J. R. y Arias Leyva, M. G. (2004). Dirección del proceso del aprendizaje de las asignaturas priorizadas: Español. En V Seminario Nacional para Educadores. La Habana: Editorial Pueblo y Educación.

Poncet, C. (1907). Lecciones de lenguaje. La Habana: Editorial La Moderna Poesía. 


\section{EDUCACIÓN}

Rico Montero, P. (1996). Reflexión y aprendizaje en el aula. La Habana: Editorial Pueblo y Educación.

Rico Montero, P. et al. (2000). Hacia el Perfeccionamiento de la Escuela Primaria.La Habana: Editorial Pueblo y Educación.

Rico Montero, P. Santos Palma, E. M. y Martín-Viaña, V (2008). Exigencias del modelo de escuela primaria para la dirección por el maestro de los procesos de educación, enseñanza y aprendizaje. La Habana: Editorial Pueblo y Educación.

Rico Montero, P. y otros (2008). Teoría de enseñanza-aprendizaje desarrollador en la escuela primaria. Teoría y práctica. La Habana: Editorial Pueblo y Educación.

Roméu Escobar, A. (1987). Metodología de la enseñanza del español. Tomo I. La Habana: Editorial Pueblo y Educación.

Roméu Escobar, A. (1999). Aplicación del enfoque comunicativo en la escuela media. En Taller de la palabra. La Habana: Editorial Pueblo y Educación.

Sales Garrido, L. (2005). La comunicación y los procesos psicológicos que influyen en la reflexión lógica y en la génesis de la comprensión y la construcción textual. En CD Maestría en Ciencias de la Educación.

Sales Garrido, L. (2007). Desarrollo de las capacidades de la comunicación oral y escrita. En Materiales Básicos de la Maestría en Ciencias de la Educación, módulo III, segunda parte. Mención en Educación Primaria. La Habana. Editorial Pueblo y Educación.

Sales Garrido, L. (1989). Obras Completas. Tomo V. La Habana: Editorial Pueblo y Educación. 